\title{
Energy Distribution Control in Wireless Sensor Networks Through Range Optimization
}

\author{
M.Sarper Gokturk, Ozgur Gurbuz and Ozgur Ercetin \\ Faculty of Engineering and Natural Sciences, Sabanci University, Istanbul 34956, Turkey \\ msgokturk@su.sabanciuniv.edu, ogurbuz@sabanciuniv.edu, oercetin@sabanciuniv.edu
}

\begin{abstract}
A major objective in wireless sensor networks is to find optimum routing strategies for energy efficient use of nodes. Routing decision and transmission power selection are intrinsically connected since the transmission power of a node is adjusted depending on the location of the next hop. In this paper, we propose a location-based routing framework to control the energy distribution in a network where transmission ranges, hence powers, of nodes are determined based on their locations. We show that the proposed framework is sufficiently general to investigate the minimum-energy and maximum-lifetime routing problems. It is shown that via the location based strategy the network lifetime can be improved by $70 \%$ and the total energy consumption can be decreased to three-fourths to one-third of the constant transmission range strategy depending on the propagation medium and the size of the network.
\end{abstract}

\section{INTRODUCTION}

Wireless sensor networks (WSN) consist of small nodes with sensing, computation and wireless communication capabilities. Each sensor node collects and routes data either to other sensor nodes or to a base station (BS), which is a node to connect the sensor network to an existing communications network. Despite innumerable applications of WSNs [1], these networks have restrictions, such as limited energy supply and limited computing power, which necessitates careful energy management strategies. Two main sources of energy consumption at a wireless node are the transceiver circuitry and the transmit amplifier. Considering a single wireless sensor node, the energy spent in the transmit amplifier is diminished by short range transmissions, while transceiver circuit energy is not affected by the transmission range. However, when the energy expenditure of the entire network is considered, lower transmission ranges result in increased number of packets to be forwarded in the network, which in turn increases the energy consumption in the transceiver circuitries. Although higher transmission powers result in higher amplifier energy consumptions, number of forwarded packets is reduced, which in turn results in lower transceiver circuit energy consumption. Therefore, it is essential to consider both the number of packets forwarded in the network and the transceiver energy consumption while designing routing strategies.

There have been intensive research to design routing strategies to achieve data communication while trying to prolong the lifetime of the network and prevent connectivity degradation by employing energy management techniques [2]. One

This work was in part supported by TUBITAK Career Grant No: 105E093 and TUBITAK Grant No:105E192. approach is to minimize the total consumed energy to reach the destination (BS) by minimizing the energy consumed per unit flow or packet [3], [4], [5]. Another approach is to maximize the lifetime of the system, which is considered as maximizing the time to network partition or the time the first node dies [6], [7]. Unlike other wireless networks, in a WSN, sensed data is usually required to be paired with the location information [2]. With the current advances in location services [8], [9], position-based approaches emerge as appropriate candidates for routing in WSNs, since they do not require establishment and maintenance of routes [10]. However, the aforementioned routing methods require current network state information such as residual energy information for correct operation [6] and incur messaging overhead on the network [4]. Obtaining accurate network state information in terms of residual energies of the nodes requires continuous and periodic updates throughout the network, which also consumes energy, and may obliterate the benefits provided by the routing algorithm. Furthermore, in wireless sensor networks, reliable data transfer while consuming the least amount of energy is of paramount importance. Applications such as habitat monitoring require reliable and predictable data reporting with varying duty cycles [11], [12]. A rule of thumb for achieving predictable operation in a network of limited capacity nodes, such as a WSN, is to reduce the complexity of communication as much as possible.

In this paper, we propose a simple routing framework for energy management in WSNs, which provides means to control the energy distribution in a network without incurring control message updates. In the proposed framework, the transmission range of each node is determined by a function of the node location. We show that by appropriate selection of the parameters of the transmission range function one can obtain simple routing solutions to minimum-energy and maximum-lifetime routing problems. As compared to constant transmission range minimum-energy strategy, the proposed framework is shown to provide significant improvements, $70 \%$, in network-lifetime when applied with the maximumlifetime objective, and reduction in energy consumption by a factor of more than two when used with the minimum energy objective. Sometimes, due to geographical limitations, the nodes in certain parts of the network may not be suitable for battery renewal as the other regions of the network. In that case, one might want to force the nodes in specific regions of the network to route their packets in such a way 


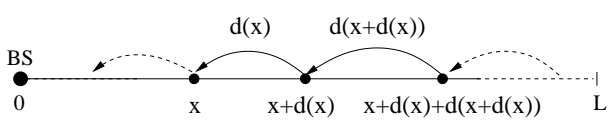

Fig. 1. Linear Network of length $L$ with the BS at the leftmost position

that the vulnerable regions are not employed for relaying excessive data for the other nodes. Routing strategies should be configured to take into account these issues and provide solutions for adjusting the energy distribution in the network. We demonstrate that our routing framework also provides a simple routing solution such that the regions that spend less or more energy can be adjusted.

\section{ROUTING FRAMEWORK}

We consider a linear network, where the base station (BS) is located at the leftmost position of the topology. The distance between the BS and the farthest node is $L$ units. Each node senses the environment and generates data to be forwarded to the BS. We assume that the nodes transmit data at a rate of 1 bit per second. The received power decays exponentially as a function of the distance, $d$, between the transmitter and the receiver according to a path loss coefficient, $\alpha$. In many practical cases, the path loss coefficient takes values $2 \leq \alpha \leq$ 4 [13].

In this work, we argue that we can control the energy distribution in the network by adjusting the transmission powers of the nodes according to the transmission range defined by a distance function, $d(x)$, where $x$ designates the location of the receiver node. Throughout this paper, without loss of generality, we denote the node at location $x$ as node $x$. As depicted in Fig. 1, node $x$ is the relay for node $x+d(x)$; node $x+d(x)$ is the relay for node $x+d(x)+d(x+d(x))$; and so on. In [14], the authors have proposed the use of a linear transmission range function to balance the energy consumption in the network; however here our goal is to provide a routing framework that enables not only minimum-energy and maximum-lifetime routing solutions but also a simple way to control the energy distribution in the network. For this purpose we propose the use of a symmetric function, whose mean, decay and increase rates and slopes can be adjusted, such as Lorentzian:

$$
d(x)=\frac{\left(\frac{\Gamma}{2}\right)^{2}}{(x-\mu)^{2}+\left(\frac{\Gamma}{2}\right)^{2}} d_{m},
$$

where $d_{m}$ is the maximum value, $\mu$ designates the center point that the function reaches $d_{m}$, i.e. $d(\mu)=d_{m}$, and $\Gamma$ designates the width of the function, i.e. $d\left(\mu \mp \frac{\Gamma}{2}\right)=\frac{d_{m}}{2}$. Let $x_{0}, x_{1}, \ldots, x_{k}$ denote the set of nodes on a particular route to the BS. As shown in Fig. 2, $x_{0}$ is the relay for node $x_{1}=x_{0}+d\left(x_{0}\right)$. Therefore, node $x_{0}$ forwards not only the data generated by itself but also the data incoming from $x_{1}$. In a similar fashion, $x_{1}$ relays the data coming from node $x_{2}=x_{1}+d\left(x_{1}\right)$. In general, node $x_{0}$ is the relay for all nodes with $x_{k}=x_{k-1}+d\left(x_{k-1}\right)$, and

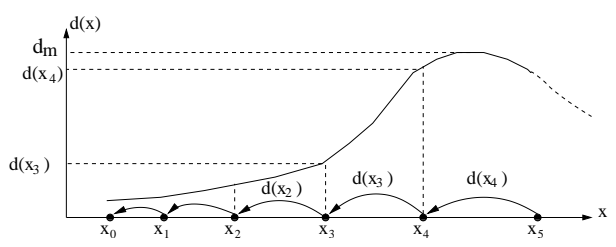

Fig. 2. Transmission distance function, $d(x)$

the location of node $x_{k}$ can be obtained as

$$
x_{k}=x_{0}+\sum_{i=0}^{k-1} \frac{\left(\frac{\Gamma}{2}\right)^{2}}{\left(x_{i}-\mu\right)^{2}+\left(\frac{\Gamma}{2}\right)^{2}} d_{m},
$$

where $k=1, \ldots, K\left(x_{0}\right)$, and $K\left(x_{0}\right)$ represents the maximum number of nodes for which $x_{0}$ acts as a relay. Since each node in the network generates one data packet, node $x_{0}$ forwards a total of $K\left(x_{0}\right)+1$ packets. The transmission range of node $x_{k}$ in terms of its own location, $d\left(x_{k}\right)$, can be found via equation (1) and by substituting $x_{k}$ with $x_{k}=x_{k-1}+d\left(x_{k-1}\right)$, which results in

$$
\begin{aligned}
& x_{k-1}^{3}-\left(x_{k}+2 \mu\right) x_{k-1}^{2}+\left(\mu^{2}+\frac{\Gamma^{2}}{4}+2 x_{k} \mu\right) x_{k-1} \\
& -x_{k} \mu^{2}-x_{k} \frac{\Gamma^{2}}{4}+\frac{\Gamma^{2}}{4} d_{m}=0,
\end{aligned}
$$

and solving for the real root of this equation as $x_{k}^{*}$, the transmission power for node $x_{k}$ is found as $P_{0} d\left(x_{k}^{*}\right)^{\alpha}$. In summary, given a Lorentzian distance function $d(x)$, the number of forwarded packets for any node $x, K(x)$, satisfies the inequality $x_{K(x)} \leq L$, and it can be calculated through the following recursion:

$$
\sum_{i=0}^{K(x)-1} \frac{1}{\left(x_{i}-\mu\right)^{2}+\left(\frac{\Gamma}{2}\right)^{2}} \leq\left(\frac{L-x}{\left(\frac{\Gamma}{2}\right)^{2} d_{m}}\right)
$$

Given the data rate of $1 \mathrm{bit} / \mathrm{sec}$, the energy consumption at a node for transmitting one bit of data is calculated as $E_{t}=$ $P_{t}+P_{0} d^{\alpha}$, and for receiving one bit as $E_{r}=P_{r}$, where $P_{t}$ and $P_{r}$ correspond to transmit and receive circuitry power consumption, and $P_{0}$ is the reference transmit amplifier power consumption for unit distance. Consequently, the energy spent at a node to relay one bit to a distance $d$ is given by $E(d)=$ $P_{r}+P_{t}+P_{0} d^{\alpha}$, and the cumulative energy consumed by node $x$ can be calculated as:

$$
E(x)=K(x)\left[P_{r}+P_{t}+P_{0} d\left(x^{*}\right)^{\alpha}\right]+\left[P_{t}+P_{0} d\left(x^{*}\right)^{\alpha}\right],
$$

where the first term is due to the data relayed from upstream nodes and the second term is due to the data generated by node $x$. In the next section, we demonstrate the use of the proposed routing framework under different objectives, such as minimum-energy, maximum-lifetime and energy distribution control.

\section{Routing Strategies}

It is known that the multi-hop routing reduces the transmission power dramatically for long-haul transmissions compared to direct transmission [15]. However, although multi-hop 
transmission reduces the energy consumption of the nodes far away from the BS, it increases the energy consumption of the intermediate nodes. In [16], the authors investigate the problem of finding the optimal number of relays and their locations in the network so that the total relaying energy is minimized. It is shown that the total relaying energy is minimized when all hop distances are set to $\frac{L}{K}$, where $K$ is the number of hops between the source and the destination. Furthermore, the optimal relay node separation is found as $d_{\text {char }}=\sqrt[\alpha]{\frac{P_{r}+P_{t}}{P_{0}(\alpha-1)}}$, and the optimal number of hops, $K_{o p t}$, required to transmit data from a node $L$ units away from the BS is given by $\left\lceil\frac{L}{d_{c h a r}}\right\rceil$. Nonetheless, this optimization problem does not consider the cumulative transmission energy consumed by the relay nodes for carrying the data from their upstream nodes, whose number can be very large. The energy consumption in the nodes increases linearly as the BS is approached, so the nodes close to the BS drain their batteries long before the other nodes. Here, we demonstrate that our routing framework can be implemented as a valid and general solution to minimum-energy, maximum-lifetime and energy distribution control objectives.

1) Location-based Minimum-Energy Routing: We apply the proposed framework with an objective of minimizing the overall energy consumption in the network. Specifically, we look for the result of the following optimization problem:

$$
\begin{aligned}
& \min _{\Gamma, \mu, d_{m}} \sum_{x} E(x) \\
& \text { s.t. } \quad 0 \leq \mu \leq L \\
& \frac{L}{3}\left[\frac{P_{\max }}{P_{0}}\right]^{\frac{1}{2 \alpha}} \leq \Gamma \leq 6 L\left[\frac{P_{\max }}{P_{0}}\right]^{\frac{1}{2 \alpha}} .
\end{aligned}
$$

The constraints on $\mu$ and $\Gamma$ impose that the center point always lies inside the linear network and $d(x)$ does not decrease in a fast manner, causing many nodes to transmit to relatively small distances. $P_{\max }$ corresponds to the maximum available transmission power, and in particular $P_{0} d_{m}^{\alpha} \leq P_{\max }$. The optimal values $\Gamma_{o p t}, \mu_{o p t}$ and $d_{m, o p t}$ are found numerically and the resulting $d(x)$ provides the optimum ranges for minimumenergy routing.

2) Location-based Maximum-Lifetime Routing: Constant transmission ranges result in an energy distribution where the nodes closer to the BS spend more energy than the other nodes. In location-based maximum-lifetime routing, our aim is to maximize the network lifetime by minimizing the maximum energy spent in the network. Among all nodes within $0<x \leq L$, there exists at least one node $\check{x}$ for which $E(\check{x})$ is the maximum. Our objective is to minimize $E(\check{x})$ by selecting appropriate $\Gamma, \mu$ and $d_{m}$. Specifically, we formulate the optimization problem as follows:

$$
\begin{aligned}
& \min _{\Gamma, \mu, d_{m}}\left[\max _{x} E(x)\right] \\
& \text { s.t. } 0 \leq \mu \leq L \\
& \frac{L}{3}\left[\frac{P_{\max }}{P_{0}}\right]^{\frac{1}{2 \alpha}} \leq \Gamma \leq 6 L\left[\frac{P_{\max }}{P_{0}}\right]^{\frac{1}{2 \alpha}} .
\end{aligned}
$$

The optimal values $\Gamma_{o p t}, \mu_{o p t}$ and $d_{m, o p t}$ are found numerically resulting in the optimum $d(x)$ for maximum-lifetime routing.

3) Location-based Energy Distribution Control: The location-based routing framework can be applied to avoid excessive use of some regions in the network while finding the routes. The intuition is that by forcing the nodes in specific regions to transmit with longer ranges we can decrease the amount of data forwarded by the downstream nodes. Also, since the nodes in the vulnerable regions have their own packets to be forwarded, the transmission ranges of these nodes should be adjusted appropriately as well. For this purpose, we formulate the energy distribution control as an optimization problem within our framework with an objective of minimizing the energy consumption in the specified regions of the network. Given $\mu_{0}$ as the desired center of the energy distribution and $\Gamma_{0}$ as the width of that distribution, we obtain $\mu_{0}^{*}$ and $\Gamma_{0}^{*}$ by substituting $x_{k}=\mu_{0}$ and $x_{k}=\Gamma_{0}$, respectively, in equation (3), and formulate the optimization problem as:

$$
\begin{aligned}
\min _{d_{m}} & {\left[\sum_{x \leq \mu-\Gamma / 2, x \leq \mu+\Gamma / 2} E(x)\right] } \\
\text { s.t. } \quad \mu=\mu_{0}^{*} & \\
& \Gamma=\Gamma_{0}^{*} .
\end{aligned}
$$

The optimal $d_{m}$ is found numerically as $d_{m, o p t}$, resulting in the optimum $d(x)$ for the desired energy distribution.

Once the optimum $d(x)$ is computed centrally by the BS according to one of the three objectives, the parameters $\Gamma_{o p t}$, $\mu_{o p t}$ and $d_{m, o p t}$ are distributed to all nodes, and no further update is necessary unless the location of a node is changed. Each node computes its transmission range through equation (3) and solves for the transmission power. The proposed framework provides advantages for implementation. Firstly, the $\mathrm{BS}$ requires only the network span, $L$, to compute the optimum $d(x)$. Secondly, each node is required to know only its own location, which can be obtained through one of the many localization algorithms proposed in the literature [8], [9]. The continuum network assumption here implies that each transmission range corresponds to exactly one node in the network, which may not be realized in real networks. In practice, the identification of the next-hop node that is closest to the transmission range can be resolved via a neighbor discovery algorithm [17].

\section{Discussions}

In this section, we evaluate our location based routing framework under the minimum-energy, maximum-lifetime and the energy distribution control objectives in comparison to constant range minimum energy routing. The energy spent and the number of packets forwarded by each node according to three routing schemes are depicted in Fig. 3 (a) and (b), for a linear network with $L=50, \alpha=4$, and $P_{t, r}=P_{0}=1$, where $P_{t, r}=P_{t}+P_{r}$ designates the power consumption in the transceiver circuitry. As observed in Fig. 3 (a), the proposed 


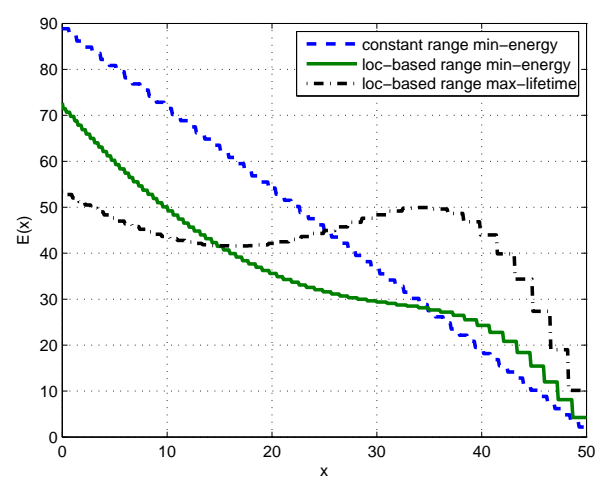

(a)

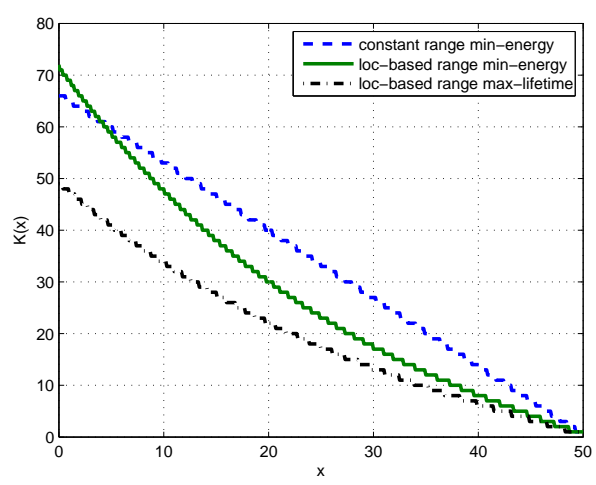

(b)

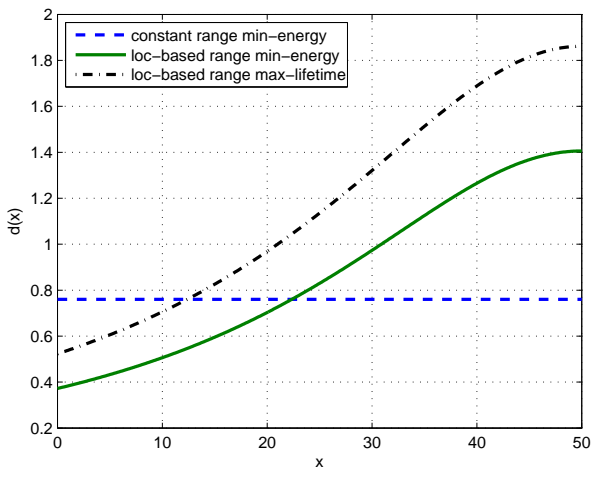

(c)

Fig. 3. Comparison of the location based range minimum-energy and maximum-lifetime, and constant range minimum-energy routing for $\alpha=4$, $L=50$, and $P_{0}=P_{r, t}=1$ : (a) Energy consumption of nodes, $E(x)$. (b) Number of packets forwarded, $K(x)$, by each node. (c) Transmission range selection for each node. The plots depict the results for the optimum range parameters: i) Constant range minimum-energy routing: $d_{c h a r}=0.7598$; ii) Location based minimum-energy routing: $\Gamma_{\text {opt }}=60, \mu_{o p t}=50, d_{m}=1.406$; iii) Location based maximum-lifetime routing: $\Gamma_{\text {opt }}=62.5, \mu_{o p t}=50, d_{m}=1.862$.

routing framework decreases the energy consumption of the nodes that are closer to the BS, while increasing the energy consumption of the nodes at the rightmost position of the linear network for both minimum-energy and maximum-lifetime criteria. Note that, the nodes at the rightmost locations have longer transmission ranges than the other nodes in the network, which is observed through the increased step sizes. As depicted in Fig. 3 (b), in location based minimum-energy routing the nodes that are very close to the BS, forward larger number of packets which is due the decreased transmission ranges of these nodes. However, although these nodes forward larger number of packets, their energy consumption is decreased by $20 \%$ as compared to constant range routing, due to the decrease in transmission ranges. The location based minimumenergy routing results in lower energy consumption in the entire network as compared to the constant range minimumenergy routing by $22 \%$, when $P_{0}=P_{t, r}=1$. When the transceiver circuit energy consumption is increased, we observe that the location based minimum-energy routing strategy provides further improvements. For instance, for the case where $P_{0}=1$ and $P_{t, r}=180$, which represents typical ratios for current radios [16], total energy savings is increased to $28 \%$. The location based routing compensates for the effect of circuit energy consumption by adjusting the transmission range appropriately, which provides energy savings compared to constant optimum range routing.

Next, we compare the maximum lifetime of networks that employ the location based and constant range routing strategies, where network lifetime is defined as the duration between the time the network begins its operation and the time the maximum energy consuming node in the network dies. It is clearly seen in Fig. 3 (a) that in all three routing strategies the nodes closer to the BS spend the maximum energy in the network and the location based routing framework with maximum-lifetime objective decreases level of maximum energy consumption in the constant range routing strategy significantly by $70 \%$, improving the lifetime by the same amount. As depicted in Fig.
3 (b), the location based maximum-lifetime strategy results in smaller number of packets to be forwarded in the network, which is due to the increased transmission ranges assigned to the nodes as observed in Fig. 3 (c). However, although the network lifetime is improved dramatically, the total energy consumption in the network is increased by the maximumlifetime strategy as a result of relatively long transmission ranges. It is interesting to note that the network lifetime of constant range minimum-energy routing can be improved by $29 \%$ by employing the location based minimum-energy routing scheme, which is also shown to provide significant energy savings in the total energy consumption.

The performance improvement of the proposed location based routing framework comes from the flexibility in assigning variable transmission ranges, which is enabled through the use of a simple distance function such as Lorentzian. The transmission ranges assigned to each node by the three routing strategies are depicted in Fig. 3 (c), where it can be seen that our location based schemes assign transmission ranges as low as half of $d_{\text {char }}$, and as high as twice $d_{\text {char }}$, for leftmost and rightmost nodes, respectively. In the location based routing framework with both objectives; i) the increased transmission ranges of the nodes away from the BS decrease the amount of packets forwarded by the nodes that are close to the BS, ii) the decreased transmission ranges assigned to the nodes closer to the BS together with the decreased number of packets to be forwarded result in lower energy consumption.

Next, we apply the location based routing strategy in an example scenario to control the energy distribution in a linear network. Suppose that the nodes in the middle of the network have plenty of power resources, while the nodes near the BS and near the edge of the network have limited power supplies. For such a case, we would like to minimize the energy consumption at the nodes near the BS and near the edge of the network, which is a quite challenging scenario for a routing problem. The nodes close to the BS have to forward packets more frequently than the other nodes in the network, 


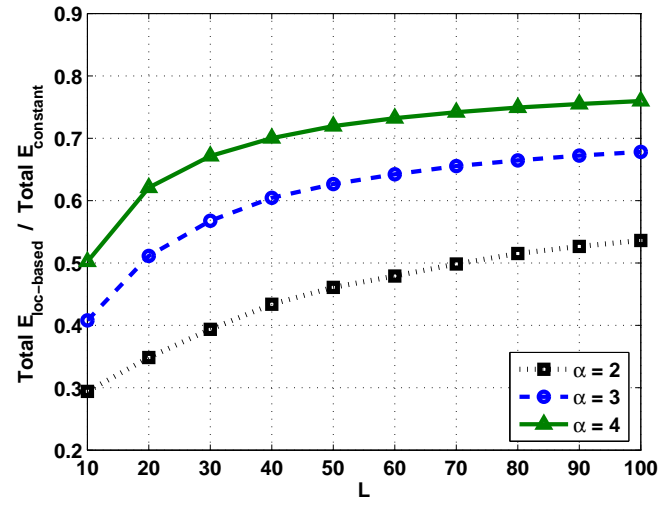

Fig. 4. The ratio of the total energy consumption in location-based minimumenergy and constant range minimum-energy routing for varying network sizes, $L$, and for $\alpha=2,3,4, P_{0}=1$ and $P_{r, t}=180$.

and in order to decrease the energy consumption of closer nodes, the farmost nodes need to transmit with higher powers increasing their own energy consumption. We formulate the location based routing when the objective is to minimize the amount of total energy consumed by the nodes located in the regions $0 \rightarrow\left[\mu-\frac{\Gamma}{2}\right]$ and $\left[\mu+\frac{\Gamma}{2}\right] \rightarrow L$, where $\mu=25$ and $\Gamma=25$. For the optimal solution of $\mu$ and $\Gamma$ that provide the range function, it is observed that the total energy consumed in the leftmost and rightmost nodes is decreased significantly by $21 \%$ as compared to constant range minimum-energy routing. This solution also corresponds to $10 \%$ energy savings with respect to the location based minimum-energy routing scheme for the designated region.

Finally, we compare the total energy consumption in location based minimum-energy and constant range minimumenergy routing schemes in different propagation environments. In Fig. 4, we depict the ratio of total energy consumption in the network using two strategies for $P_{0}=1, P_{t, r}=180$, varying $L$, and $\alpha=2,3,4$. It is observed that energy savings of at least $25 \%$ is obtained and depending on the path-loss coefficient, energy consumption of the location based strategy can be as low as one third of the constant range minimumenergy routing.

\section{CONCLUSiOns}

In this work, we propose a general location-based routing framework that controls the energy distribution of a wireless sensor network via transmission range optimization. The idea presented here is not limited to the use in WSNs, and is general enough to apply for other wireless networks, particularly relay networks as well. The location based range optimization framework is shown to be well suited for minimum-energy and maximum-lifetime routing problems and for a general energy distribution control objective. It is shown that the location based minimum-energy routing scheme results in 2 to 3 times less energy consumption in the network as compared to constant range minimum-energy routing. Also, the location based framework with the maximum-lifetime objective is shown to improve the network lifetime by $70 \%$ as compared to the constant range minimum-energy routing strategy. Since the range of a node does not change as long as its location is not changed, our framework eliminates the need for exchanging residual energy information as opposed to previously proposed routing algorithms in the literature. In short, our framework provides a simple way to control the energy distribution in the network making it a practical routing alternative for WSN applications.

\section{REFERENCES}

[1] I. F. Akyildiz, W. Su, Y. Sankarasubramaniam, and E. Cayirci, "A Survey on Sensor Networks," IEEE Commun. Mag., vol. 40, pp. 102-114, Aug. 2002.

[2] J. N. Al-Karaki and A. E. Kamal, "Routing Techniques in Wireless Sensor Networks: A Survey," IEEE Wireless Commun., vol. 11, no. 6, pp. 6-28, Dec. 2004.

[3] P. Chen, B. O'Dea, and E. Callaway, "Energy Efficient System Design with Optimum Transmission Range for Wireless Ad hoc Networks," Proc. IEEE ICC 2002, vol. 2, pp. 945-952, May 2002.

[4] Y. Xu, J. Heidemann, and D. Estrin, "Geography-informed Energy Conservation for Ad hoc Routing," Proc. ACM/IEEE MobiCom 2001, pp. 70-84, July 2001.

[5] V. Rodoplu and T. H. Meng, "Minimum Energy Mobile Wireless Networks," IEEE J. Sel. Areas Commun., vol. 17, no. 8, Aug. 1999.

[6] J.-H. Chang, L. Tassiulas, "Energy Conserving Routing in Wireless Adhoc Networks," Proc. IEEE INFOCOM 2000, vol. 1, pp. 22-31, March 2000.

[7] A. Sankar and Z. Liu, "Maximum Lifetime Routing in Wireless Ad-hoc Networks," Proc. IEEE INFOCOM 2004, vol. 2, pp. 1089-1097, March 2004.

[8] N. Patwari, J.N. Ash, S. Kyperountas, A.O. Hero III, R.L. Moses, N.S Correal, "Locating the Nodes: Cooperative Localization in Wireless Sensor Networks," IEEE Signal Process. Mag., vol. 22, no. 4, pp. 54-69, July 2005.

[9] N. Bulusu, J. Heidemann, and D. Estrin, "GPS-less Low-Cost Outdoor Localization for Very Small Devices," IEEE Pers. Commun. Mag., vol.7, no.5, pp. 28-34, Oct. 2000.

[10] M. Mauve, J. Widmer, H. Hartenstein, "A Survey on Position-based Routing in Mobile Ad Hoc Networks," IEEE Network, vol. 15, no. 6, pp. 30-39, Nov/Dec 2001.

[11] A. Mainwaring, J. Polastre, R. Szewczyk, D. Culler, J. Anderson, "Wireless Sensor Networks for Habitat Monitoring," ACM WSNA'02, pp. 88-97, Sep. 2002.

[12] A. Cerpa, J. Elson, M. Hamilton, J. Zhao, "Habitat Monitoring: Application Driver for Wireless Communications Technology," ACM SIGCOMM, vol. 31, pp. 20-41, April 2001.

[13] T. S. Rappaport, Wireless Communications: Principles and Practice, 2nd ed. Upper Saddle River, NJ: Prentice Hall, 2002.

[14] O. Ercetin, "Distance-Based Routing for Balanced Energy Consumption in Sensor Networks," preprint.

[15] W. R. Heinzelman, A. Chandrakasan, and H. Balakrishnan, "EnergyEfficient Communication Protocol for Wireless Microsensor Networks,' Proc. 33rd Hawaii Int. Conf. on System Sciences, vol. 2, pp. 1-10, Jan. 2000.

[16] M. Bhardwaj, T. Garnett, and A. Chandrakasan, "Upper Bounds on the Lifetime of Sensor Networks," Proc. IEEE ICC 2001, vol. 3, pp. 785790, June 2001

[17] S. A. Borbash, A. Ephremides, M. J. McGlynn, "An Asynchronous Neighbor Discovery Algorithm for Wireless Sensor Networks," ACM Ad hoc Networks Journal, vol. 5, no.7, pp. 998-1016, Sep. 2007. 